PROCEEDINGS OF THE

AMERICAN MATHEMATICAL SOCIETY

Volume 134, Number 11, November 2006, Pages 3211-3221

S 0002-9939(06)08358-4

Article electronically published on May 11, 2006

\title{
FURTHER RESULTS ON THE CONNECTIVITY OF PARSEVAL FRAME WAVELETS
}

\author{
G. GARRIGÓS, E. HERNÁNDEZ, H. ŠIKIĆ, AND F. SORIA
}

(Communicated by David R. Larson)

\begin{abstract}
In a previous paper, the authors introduced new ideas to treat the problem of connectivity of Parseval frames. With these ideas it was shown that a large set of Parseval frames is arcwise connected. In this article we exhibit a larger class of Parseval frames for which the arcwise connectivity is true. This larger class fails to include all Parseval frames.
\end{abstract}

\section{INTRODUCTION AND MAIN RESUlt}

The connectivity on the set of orthonormal wavelets is an interesting problem which remains open many years after it was proposed (see for instance [1, 10, 9, 5]). The question has been extended to the larger class of Parseval frame wavelets (see [7] and [3). Even in this extended setting, the problem seems very difficult and is still open. We recall that a function $\psi \in L^{2}(\mathbb{R})$ is said to be a Parseval frame wavelet (or a normalized tight frame wavelet) if the collection $\left\{\psi_{j, k}(x)=\right.$ $\left.2^{j / 2} \psi\left(2^{j} x-k\right): j \in \mathbb{Z}, k \in \mathbb{Z}\right\}$ forms a tight frame (with constant 1 ) for $L^{2}(\mathbb{R})$. When this is the case we shall write $\psi \in \mathbf{P F W}$. It is well known (see [4, Ch.7]) that these functions are characterized by the two equations:

$$
\begin{array}{ll}
\sum_{j \in \mathbb{Z}}\left|\widehat{\psi}\left(2^{j} \xi\right)\right|^{2}=1 & \text { a.e. } \xi \in \mathbb{R}, \\
t_{q}(\xi ; \psi):=\sum_{j=0}^{\infty} \widehat{\psi}\left(2^{j} \xi\right) \overline{\widehat{\psi}\left(2^{j}(\xi+2 q \pi)\right)}=0 & \text { a.e. } \xi \in \mathbb{R}, \quad \forall q \in 2 \mathbb{Z}+1 .
\end{array}
$$

This characterization is used in 3] to give a new approach to the connectivity of the set PFW. In particular, in that paper we were able to construct explicit paths for two large subclasses $\mathbf{K}_{\tau}$ and $\mathbf{K}_{d}$ of $\mathbf{P F W}$, defined by size conditions on the spectrum (see section 3 below). Since each subclass in some sense takes care of one of the basic transformations (translations and dilations), one may conjecture that finding a condition that encompasses both would settle the problem completely. Unfortunately, as we shall prove in this article, this is not necessarily so. We

Received by the editors January 24, 2005 and, in revised form, May 12, 2005.

2000 Mathematics Subject Classification. Primary 42C15, 42C40.

Key words and phrases. Connectivity, tight frames, Parseval frames.

The first, second and fourth authors were supported by grant MTM2004-0678, MEC (Spain). The third author was supported by grants MZOŠ 0037118 (Croatia) and USA-Croatia NSF INT0245238. The first author was also supported by Programa Ramón y Cajal, 2001, MCyT (Spain). 
provide a new condition along that direction which defines an even larger class of connected Parseval frame wavelets, but that it does not include the entire PFW.

We shall say that $J \subset \mathbb{R}$ is a Calderón set if the family $\left\{2^{j} J\right\}_{j \in \mathbb{Z}}$ forms an a.e. partition of $\mathbb{R} \backslash\{0\}$. A particular example is the Shannon set $S=[-2 \pi,-\pi) \cup(\pi, 2 \pi]$. Observe that $J$ is a Calderón set if and only if the function $\hat{\psi}=\chi_{J}$ satisfies (A1). By (A2), if in addition $J \subset[-\pi, \pi]$, then $\psi \in \mathbf{P F W}$. For sets $A, K \subset \mathbb{R}$ we shall also use the following notation from [3]:

$$
[A]=\bigcup_{j \in \mathbb{Z}} 2^{-j} A, \quad[A]_{-}=\bigcup_{j=0}^{\infty} 2^{-j} A, \quad \text { and } \quad \tau_{K}(A)=\bigcup_{\substack{k \in \mathbb{Z} \\ k \neq 0}}(A+2 k \pi) \cap K .
$$

Definition 1.1. Let $J_{0}$ be a bounded Calderón set and denote $J_{j}=2^{-j} J_{0}, j \in \mathbb{Z}$. We say that a measurable subset $K \subset \mathbb{R}$ is $J_{0}$-admissible or that it belongs to the class $\mathcal{K}\left(J_{0}\right)$ whenever there exists $M \in \mathbb{Z}$ such that the set correspondence

$$
I \subset\left[J_{M}\right]_{-}=\bigcup_{\ell=M}^{\infty} 2^{-\ell} J_{0} \longmapsto \mathcal{L}(I)=\mathcal{L}_{K}(I)=\left[\tau_{K}(I)\right] \cap S
$$

is continuous in the sense of the measure algebras. That is, for each $\eta>0$ there exists $\delta>0$, such that $\forall I \subset\left[J_{M}\right]_{-}$with $|I|<\delta$, we have $|\mathcal{L}(I)|<\eta$.

It is easy to see that bounded sets belong to the class $\mathcal{K}(S)$. Indeed, in such a case only finite unions of translates and dilates of $I$ are involved in the definition of $\mathcal{L}_{K}(I)$, whenever $I \subset(-\pi, \pi)$. The continuity then follows easily by subadditivity. It is also easy to see from the definition that $\mathcal{K}(S) \subset \mathcal{K}\left(J_{0}\right)$ for any bounded Calderón set $J_{0}$. Indeed, the "domain" of the set correspondence in Definition 1.1 is always of the form $(-\varepsilon, \varepsilon)$ for the class $\mathcal{K}(S)$, while for general classes $\mathcal{K}\left(J_{0}\right)$ it is only a subset of one such interval. We do not know whether $\mathcal{K}(S)=\mathcal{K}\left(J_{0}\right)$ for general $J_{0}$.

Concerning the connectivity of PFW, the main result in this paper is the following.

Theorem 1.2. Let $J_{0} \subset[-\pi, \pi]$ be a Calderón set. Then, for every $\psi \in \mathbf{P F W}$ such that Supp $\widehat{\psi}$ belongs to the class $\mathcal{K}\left(J_{0}\right)$, there exists a continuous path $\left\{\psi_{t}\right\}_{0 \leq t \leq 1}$ inside PFW such that

$$
\psi_{0}=\psi, \quad \psi_{1}=\left(\chi_{J_{0}}\right)^{\smile} \quad \text { and } \quad \operatorname{Supp} \widehat{\psi}_{t} \subset \operatorname{Supp} \widehat{\psi} \cup\left[J_{0}\right]_{-} .
$$

In particular, the set of all $\psi \in \mathbf{P F W}$ with Supp $\widehat{\psi} \in \mathcal{K}\left(J_{0}\right)$ is arcwise connected in $L^{2}(\mathbb{R})$.

Since bounded Calderón sets belong to $\mathcal{K}(S)$, Theorem 1.2 applied to $\widehat{\psi}=\chi_{J_{0}}$ shows that $\check{\chi}_{J_{0}}$ and $\check{\chi}_{S}$ can be continuously connected within PFW. Thus, as a corollary we obtain that the class of all PFW's with spectrum in $\mathcal{K}\left(J_{0}\right)$ for some $J_{0}$ is arcwise connected. Moreover, the proof of Theorem 1.2 shows that the subclass of MSF Parseval frames is arcwise connected, recovering in a different way a result from 7 .

The paper is structured as follows. The proof of Theorem 1.2 is given in section 2. In section 3 we show the relation between the class $\mathcal{K}(S)$ and the classes $\mathcal{K}_{\tau}$ and $\mathcal{K}_{d}$ from the previous paper 3 . Finally, in sections 4 and 5 we show that $\mathbb{R} \notin \mathcal{K}\left(J_{0}\right)$ for every Calderón set $J_{0}$, as well as the connections of this result with number theory. 


\section{The PROOF OF THE THEOREM}

The following elementary properties about the class $\mathcal{K}\left(J_{0}\right)$ are easily verified:

(1) For any $j \in \mathbb{Z}, \mathcal{K}\left(J_{0}\right)=\mathcal{K}\left(2^{j} J_{0}\right)$. Thus, there is no loss in assuming $J_{0} \subset[-\pi, \pi]$.

(2) If $K_{1}, K_{2}$ belong to $\mathcal{K}\left(J_{0}\right)$, then so do $K_{1} \cup K_{2}$ and any subset of $K_{1}$ or $K_{2}$.

(3) In Definition 1.1, the Shannon set $S$ can be replaced by any Calderón set $H$, provided it has finite measure. More precisely, given any fixed set $E$ in $\mathbb{R}$ the map

$$
I \subset E \longmapsto \mathcal{L}^{(H)}(I):=\left[\tau_{K}(I)\right] \cap H
$$

is continuous if and only if $I \subset E \mapsto \mathcal{L}^{(S)}(I)$ is continuous. This is an elementary consequence of the following lemma.

Lemma 2.1. Let $H_{1}, H_{2}$ be two Calderón sets with finite Lebesgue measure. Then, $\forall \eta>0, \exists \delta>0$ such that $\forall A \subset \mathbb{R}:\left|[A] \cap H_{1}\right|<\delta \Longrightarrow\left|[A] \cap H_{2}\right|<\eta$.

Proof. Define the set function: $\mu(F)=\left|[F] \cap H_{2}\right|, F \subset H_{1}$. From the Calderón property of $H_{1}$ one easily checks that $\mu$ is a well-defined measure in $H_{1}$. Moreover, it is clear that $\mu$ is finite and absolutely continuous with respect to the Lebesgue measure $d x_{\left.\right|_{H_{1}}}$. Then, the usual characterization of absolutely continuous measures applied to $F=[A] \cap H_{1}$ establishes the lemma (see [8, Th.6.11]).

Proof of Theorem 1.2. Let $\psi \in \mathbf{P F W}$ be so that its spectrum, $K=\operatorname{Supp} \widehat{\psi}$, is $J_{0}$-admissible. Let us choose a large positive integer $M=M\left(K, J_{0}\right)$ so that the map $I \subset\left[J_{M}\right]_{-} \mapsto \mathcal{L}_{K}(I)$ is continuous (by the $\mathcal{K}\left(J_{0}\right)$ condition). Since $J_{0}$ and $J_{M}=2^{-M} J_{0}$ are trivially connected with the continuous path $\left\{t J_{0}\right\}_{t \in\left[2^{-M}, 1\right]}$, we may assume through the rest of the proof that $M=0$. Following the ideas in [3], we divide the proof in three parts.

Step 1 (Construction of new PFW's). As in [3], we present a general procedure to construct new PFW's starting from a given one $\psi$ and an arbitrary interval $I$ in $J_{0}$. The dynamics of the construction is the following: suppose $I=I_{0}$ is an arbitrary subset of $J_{0}$, hence contained in $[-\pi, \pi]$. Construct by induction the following sequence of subsets of $[-\pi, \pi]$ :

$$
\begin{array}{rcc}
I_{1} & = & \left(\left[\tau_{K}\left(I_{0}\right)\right] \backslash\left[I_{0}\right]\right) \cap J_{1} \\
I_{2} & = & \left(\left[\tau_{K}\left(I_{1}\right)\right] \backslash\left[I_{0} \cup I_{1}\right]\right) \cap J_{2}, \\
\vdots & \vdots \\
I_{N+1} & = & \left(\left[\tau_{K}\left(I_{N}\right)\right] \backslash\left[I_{0} \cup \ldots \cup I_{N}\right]\right) \cap J_{N+1} .
\end{array}
$$

Define $E_{I}=\bigcup_{N=0}^{\infty} I_{N}$ and note that by construction this union is disjoint (we shall denote this by the symbol $\biguplus$ ). Moreover, using the fact

$$
\left[I_{\ell}\right]=\biguplus_{j \in \mathbb{Z}} 2^{j} I_{\ell}=\left[\tau_{K}\left(I_{\ell-1}\right)\right] \backslash\left(\bigcup_{N=0}^{\ell-1}\left[I_{N}\right]\right),
$$

it is easy to verify that:

(1) $\left[E_{I}\right]=\bigcup_{\ell=0}^{\infty}\left[I_{\ell}\right]$, with disjoint union;

(2) $\left\{2^{j} E_{I}\right\}_{j \in \mathbb{Z}}$ is a disjoint family;

(3) $\left[\tau_{K}\left(E_{I}\right)\right]=\bigcup_{N=0}^{\infty}\left[\tau_{K}\left(I_{N}\right)\right] \subset \bigcup_{N=0}^{\infty}\left(\bigcup_{\ell=0}^{N+1}\left[I_{\ell}\right]\right)=\left[E_{I}\right]$. 
Since we also have $E_{I} \subset[-\pi, \pi]$, we conclude from Proposition 2.1 of $[3$ that $\psi_{E_{I}} \in \mathbf{P F W}$, where $\widehat{\psi}_{E_{I}}(\xi)=\widehat{\psi}(\xi)$ if $\xi \in \mathbb{R} \backslash\left[E_{I}\right]$, and $\widehat{\psi}_{E_{I}}(\xi)=\chi_{E_{I}}(\xi)$ if $\xi \in\left[E_{I}\right]$.

Step 2 (Construction of the path). Since $J_{0}$ is a Calderón set, there is a bijective measurable mapping $\Gamma: \xi \in J_{0} \longmapsto 2^{j(\xi)} \xi \in S$. Define the following family of subsets of $J_{0}: I(t)=\Gamma^{-1}(\{\pi<|\xi| \leq(1+t) \pi\}), t \in[0,1]$. Observe that $I(0)=\emptyset$ and $I(1)=J_{0}$. Consider the family $\psi_{t}=\psi_{E_{I(t)}}$ inside PFW obtained from the sets $E_{I(t)}$ as in the previous step. Observe that

$$
\widehat{\psi}_{t}=\widehat{\psi}_{E_{I(t)}}= \begin{cases}\widehat{\psi}, & \text { if } t=0, \\ \chi_{J_{0}}, & \text { if } t=1,\end{cases}
$$

while Supp $\widehat{\psi}_{t} \subset\left(\operatorname{Supp} \widehat{\psi} \backslash\left[E_{I(t)}\right]\right) \cup E_{I(t)} \subset \operatorname{Supp} \widehat{\psi} \cup\left[J_{0}\right]_{-}$. Finally, since bounded sets always belong to $\mathcal{K}\left(J_{0}\right)$, we have $\left[J_{0}\right]_{-} \subset[-\pi, \pi] \in \mathcal{K}\left(J_{0}\right)$. Therefore, using Supp $\widehat{\psi} \in \mathcal{K}\left(J_{0}\right)$ and the properties at the beginning of section 2 , we conclude that Supp $\widehat{\psi}_{t} \in \mathcal{K}\left(J_{0}\right)$. Thus, we have constructed a path $t \in[0,1] \mapsto \psi_{t} \in \mathbf{P F W}$ with the properties required in the theorem, and it only remains to show the $L^{2}(\mathbb{R})$ continuity of such a path.

Step 3 (Continuity of the path). By Proposition 2.2 of 3 , a path of the form $\left\{\psi_{E(t)}\right\}_{0 \leq t \leq 1}$ inside PFW is continuous provided we can show

$$
\lim _{\substack{t \rightarrow t_{0} \\ t \in[0,1]}}\left|E(t) \Delta E\left(t_{0}\right)\right|=\lim _{\substack{t \rightarrow t_{0} \\ t \in[0,1]}}\left|\widetilde{E}(t) \Delta \widetilde{E}\left(t_{0}\right)\right|=0, \quad \forall t_{0} \in[0,1],
$$

where $\widetilde{E}=[E] \cap S, E \subset \mathbb{R} \backslash\{0\}$. Now, observe that when $t \searrow t_{0}$ we have

$$
\left|I(t) \Delta I\left(t_{0}\right)\right|=\left|\Gamma^{-1}\left(\left\{\left(1+t_{0}\right) \pi<|\xi| \leq(1+t) \pi\right\}\right)\right| \longrightarrow\left|\Gamma^{-1}(\emptyset)\right|=0,
$$

and when $t \nearrow t_{0}$ we have

$$
\left|I(t) \Delta I\left(t_{0}\right)\right|=\left|\Gamma^{-1}\left(\left\{(1+t) \pi<|\xi| \leq\left(1+t_{0}\right) \pi\right\}\right)\right| \rightarrow\left|\Gamma^{-1}\left(\left\{|\xi|=\left(1+t_{0}\right) \pi\right\}\right)\right|=0
$$

in this last case since $\Gamma^{-1}$ preserves null sets. Thus, for the continuity of our path $t \rightarrow \psi_{t}$ it suffices to show that

$$
\left|E_{I} \Delta E_{I^{\prime}}\right|+\left|\widetilde{E}_{I} \Delta \widetilde{E}_{I^{\prime}}\right| \longrightarrow 0, \quad \text { as }\left|I \Delta I^{\prime}\right| \rightarrow 0 .
$$

We shall deduce this from our key assumption: $K=\operatorname{Supp} \widehat{\psi} \in \mathcal{K}\left(J_{0}\right)$. That is, the set mapping $I \subset\left[J_{0}\right]_{-} \mapsto \mathcal{L}(I)$ is continuous in the sense of measure algebras. Now, by continuity, for every $\eta>0$ there exists an integer $M_{0}=M_{0}(\eta) \geq 0$ such that

$$
\left|\left[\tau_{K}\left(\left[J_{0}\right]_{-} \cap\left(-\frac{\pi}{2^{M_{0}}}, \frac{\pi}{2^{M_{0}}}\right)\right)\right] \cap S\right|<\eta
$$

We may assume that $M_{0}$ is large enough so that $\left|\left(-\frac{\pi}{2^{M_{0}}}, \frac{\pi}{2^{M_{0}}}\right)\right|=\frac{2 \pi}{2^{M_{0}}}<\eta$. Therefore, for all $M \geq M_{0}$, and for all $I \subset J_{0}$ we have

$$
\begin{gathered}
\bigcup_{N=M}^{\infty} I_{N} \subset\left[J_{0}\right]_{-} \cap\left(-\frac{\pi}{2^{M_{0}}}, \frac{\pi}{2^{M_{0}}}\right) \quad \text { and } \\
\bigcup_{N=M+1}^{\infty} \widetilde{I}_{N}=\bigcup_{N=M}^{\infty}\left[I_{N+1}\right] \cap S \subset \bigcup_{N=M}^{\infty}\left[\tau_{K}\left(I_{N}\right)\right] \cap S=\left[\tau_{K}\left(\bigcup_{N=M}^{\infty} I_{N}\right)\right] \cap S=\mathcal{L}\left(\bigcup_{N=M}^{\infty} I_{N}\right) .
\end{gathered}
$$


Thus,

$$
\left|\bigcup_{N=M+1}^{\infty} I_{N}\right|+\left|\bigcup_{N=M+1}^{\infty} \widetilde{I}_{N}\right|<2 \eta, \quad \forall M \geq M_{0}
$$

Let us now show that the second term in the sum (2.3) converges to 0. Observe that, for all $M \geq 0$,

$$
\begin{aligned}
\widetilde{E}_{I} \Delta \widetilde{E}_{I^{\prime}} & =\left(\bigcup_{N=0}^{\infty} \widetilde{I}_{N}\right) \Delta\left(\bigcup_{N=0}^{\infty} \widetilde{I}_{N}^{\prime}\right) \\
& \subset \bigcup_{N=0}^{M}\left(\widetilde{I}_{N} \Delta \widetilde{I}_{N}^{\prime}\right) \cup\left(\bigcup_{N=M+1}^{\infty} \widetilde{I}_{N}\right) \cup\left(\bigcup_{N=M+1}^{\infty} \widetilde{I}_{N}^{\prime}\right) .
\end{aligned}
$$

Therefore, if we are able to show that for $I, I^{\prime} \subset J_{0}$

$$
\left|\widetilde{I}_{N} \Delta \widetilde{I}_{N}^{\prime}\right| \longrightarrow 0, \text { as }\left|I \Delta I^{\prime}\right| \rightarrow 0, \quad \forall N=0,1,2, \ldots,
$$

then, combining (2.5) with (2.4) it will follow that $\left|\widetilde{E}_{I} \Delta \widetilde{E}_{I^{\prime}}\right| \rightarrow 0$, as $\left|I \Delta I^{\prime}\right| \rightarrow 0$. Now, to show (2.6) we first consider the case $N=0$ : since $I, I^{\prime} \subset J_{0}$ we have

$$
\widetilde{I} \Delta \widetilde{I}^{\prime}=\left([I] \Delta\left[I^{\prime}\right]\right) \cap S=\left[I \Delta I^{\prime}\right] \cap S .
$$

Then, by Lemma 2.1 the measure of this set goes to 0 as $\left|I \Delta I^{\prime}\right|=\mid\left[I \Delta I^{\prime}\right] \cap$ $J_{0} \mid \rightarrow 0$. For the general case we proceed as in Lemma 4.4 of [3] : write $\widetilde{I}_{N+1}=$ $\left[I_{N+1}\right] \cap S=\left(\left[\tau_{K}\left(I_{N}\right)\right] \backslash\left[\bigcup_{\ell=0}^{N} I_{\ell}\right]\right) \cap S$, and use standard Boolean algebra to obtain $\widetilde{I}_{N+1} \Delta \widetilde{I}_{N+1}^{\prime} \subset\left(\left[\tau_{K}\left(I_{N} \Delta I_{N}^{\prime}\right)\right] \cup\left(\bigcup_{\ell=0}^{N}\left[I_{\ell} \Delta I_{\ell}^{\prime}\right]\right)\right) \cap S$. Then, we proceed by induction: for $N=0$ we have $\left|\widetilde{I}_{1} \Delta \widetilde{I}_{1}^{\prime}\right| \leq\left|\left[\tau_{K}\left(I \Delta I^{\prime}\right)\right] \cap S\right|+\left|\left[I \Delta I^{\prime}\right] \cap S\right|$, for which the first summand goes to 0 by the continuity assumption (as $\left|I \Delta I^{\prime}\right| \rightarrow 0, I, I^{\prime} \subset J_{0}$ ), and the second one by (2.7). Suppose now that we have shown

$$
\left|\widetilde{I}_{\ell} \Delta \widetilde{I}_{\ell}^{\prime}\right| \longrightarrow 0, \text { as }\left|I \Delta I^{\prime}\right| \rightarrow 0, \quad \forall \ell=0, \ldots, N .
$$

Again, by Lemma 2.1 we have that

$$
\left|I_{\ell} \Delta I_{\ell}^{\prime}\right|=\left|\left[I_{\ell} \Delta I_{\ell}^{\prime}\right] \cap J_{k_{\ell}}\right| \longrightarrow 0, \text { as }\left|\left[I_{\ell} \Delta I_{\ell}^{\prime}\right] \cap S\right|=\left|\widetilde{I}_{\ell} \Delta \widetilde{I}_{\ell}^{\prime}\right| \rightarrow 0 .
$$

Then, using the induction hypothesis and the continuity assumption we obtain

$$
\left|\widetilde{I}_{N+1} \Delta \widetilde{I}_{N+1}^{\prime}\right| \leq\left|\left[\tau_{K}\left(I_{N} \Delta I_{N}^{\prime}\right)\right] \cap S\right|+\sum_{\ell=0}^{N}\left|\widetilde{I}_{\ell} \Delta \widetilde{I}_{\ell}^{\prime}\right| \longrightarrow 0, \text { as }\left|I \Delta I^{\prime}\right| \rightarrow 0,
$$

establishing our claim, and concluding the proof of (2.6). To completely establish (2.3), it remains to show that $\left|E_{I} \Delta E_{I^{\prime}}\right| \rightarrow 0$ as $\left|I \Delta I^{\prime}\right| \rightarrow 0$. This follows from our previous arguments since

$$
\left|E_{I} \Delta E_{I^{\prime}}\right| \leq \sum_{N=0}^{M}\left|I_{N} \Delta I_{N}^{\prime}\right|+\left|\bigcup_{N=M+1} I_{N}\right|+\left|\bigcup_{N=M+1} I_{N}^{\prime}\right|
$$

so we just need to combine (2.4) with (2.8). 


\section{Sufficient CONDITIONS}

3.1. The $\mathcal{K}_{\tau}$ condition. We recall from [3] that a set $K \subset \mathbb{R}$ is said to belong to the class $\mathcal{K}_{\tau}$ whenever there exists $\varepsilon \in(0, \pi]$ such that

$$
\sum_{|n| \geq 1} \frac{1}{|n|}|K \cap(2 n \pi+(-\varepsilon, \varepsilon))|<\infty .
$$

Proposition 3.1. $\mathcal{K}_{\tau} \subset \mathcal{K}(S)$.

Proof. Let $K \in \mathcal{K}_{\tau}$ and choose $\varepsilon$ as in (3.1). Given $H=[-\varepsilon,-\varepsilon / 2) \cup(\varepsilon / 2, \varepsilon]$, it suffices to show that $I \subset(-\varepsilon, \varepsilon) \mapsto \mathcal{L}^{(H)}(I)$ (as in (2.1)) is continuous in measure. By Lemma 4.3 in 3 , there exists a constant $C>0$ such that for each such $I$

$$
\left|\left[\tau_{K}(I)\right] \cap H\right| \leq C \varepsilon \sum_{|n| \geq 1} \frac{1}{|n|}|K \cap(2 n \pi+I)|=C \varepsilon \int_{I} \sum_{n \geq 1} \frac{1}{|n|} \chi_{K+2 n \pi}(\xi) d \xi .
$$

Since $K \in \mathcal{K}_{\tau}$ the right-hand side of this equalities is finite. The result follows by an application of the Lebesgue dominated convergence theorem.

3.2. The $\mathcal{K}_{d}$ condition. Next we turn to the condition $\mathcal{K}_{d}$ in 3 . We say that a set $K$ belongs to such class whenever there exists $\varepsilon \in(0, \pi]$ such that

$$
\left|\limsup _{n \rightarrow \infty} \frac{1}{2^{n}}\left(K \cap 2^{n} S^{\varepsilon}\right)\right|=0, \quad \text { where } \quad S^{\varepsilon}:=[-\varepsilon,-\varepsilon / 2) \cup(\varepsilon / 2, \varepsilon] .
$$

If we define $L_{m}=L_{m}^{(\varepsilon)}=\bigcup_{n=m}^{\infty} \frac{1}{2^{n}}\left(K \cap 2^{n} S^{\varepsilon}\right)$, condition (3.2) is the same as saying that $\left|L_{m}\right| \rightarrow 0$ as $m \rightarrow \infty$. By simple Boolean algebra, $L_{m}=\left[K \cap\left(\bigcup_{n=m}^{\infty} 2^{n} S^{\varepsilon}\right)\right] \cap$ $S^{\varepsilon}$. Since $\bigcup_{n=m}^{\infty} 2^{n} S^{\varepsilon}= \pm\left(\frac{2^{m} \varepsilon}{2}, \infty\right)$, we see that (3.2) is actually equivalent to

$$
\left|[K \cap((-\infty,-R) \cup(R, \infty))] \cap S^{\varepsilon}\right| \rightarrow 0 \quad \text { as } \quad R \rightarrow \infty .
$$

Proposition 3.2. $\mathcal{K}_{d} \subset \mathcal{K}(S)$.

Proof. Let $K \in \mathcal{K}_{d}$ and $\varepsilon$ as in (3.2). Take any $\eta>0$ and $I \subset(-\varepsilon, \varepsilon)$. By (3.3), there exists $R_{0}>0$ such that

$$
\left|\left[K \cap\left(\left(-\infty,-R_{0}\right) \cup\left(R_{0}, \infty\right)\right)\right] \cap S^{\varepsilon}\right|<\frac{\eta}{2} .
$$

Let $k_{0}$ be such that $2 k_{0} \pi>R_{0}+2 \pi$, so that if $|n|>k_{0}$ we have $(2 n \pi+I) \cap K \subset$ $K \cap\left(-\infty,-R_{0}\right) \cup\left(R_{0}, \infty\right)$. Then,

$$
\begin{aligned}
& {\left[\tau_{K}(I)\right] \cap S^{\varepsilon}=\left[\bigcup_{n \in \mathbb{Z}, n \neq 0}(2 n \pi+I) \cap K\right] \cap S^{\varepsilon}} \\
& \subset\left\{\bigcup_{0<|n| \leq k_{0}}[(2 n \pi+I) \cap K] \cap S^{\varepsilon}\right\} \cup\left\{\left[K \cap\left(\left(-\infty,-R_{0}\right) \cup\left(R_{0}, \infty\right)\right)\right] \cap S^{\varepsilon}\right\} .
\end{aligned}
$$

Thus, by (3.4) and Lemma 5.6 in 3 .

$$
\left|\tau_{K}(I) \cap S^{\varepsilon}\right|<\frac{\eta}{2}+\sum_{0<|n| \leq k_{0}}\left|[(2 n \pi+I) \cap K] \cap S^{\varepsilon}\right| \leq \frac{\eta}{2}+k_{0}|I| .
$$

Choosing $\delta<\frac{\eta}{2 k_{0}}$ we obtain $\left|\tau_{K}(I) \cap S^{\varepsilon}\right|<\frac{\eta}{2}+\frac{\eta}{2}$ if $|I|<\delta$. This shows that $\mathcal{L}_{K}^{\left(S^{\varepsilon}\right)}$ is continuous and hence that $K \in \mathcal{K}(S)$. 
3.3. Other examples. The following examples show that $\mathcal{K}_{\tau} \cup \mathcal{K}_{d} \subset \mathcal{K}(S)$, that is, there exist sets $K$ which are admissible according to Definition 1.1, but are not contained in the setting of our previous paper [3]. In fact, it will suffice to consider $K=K_{1} \cup K_{2}$, where $K_{1} \in \mathcal{K}_{\tau} \backslash \mathcal{K}_{d}$ and $K_{2} \in \mathcal{K}_{d} \backslash \mathcal{K}_{\tau}$. Indeed, when this is the case, we necessarily have that $K \notin \mathcal{K}_{\tau} \cup \mathcal{K}_{d}$, while from the second observation at the beginning of section 2 (and Propositions 3.1 and 3.2) we see that $K \in \mathcal{K}(S)$.

Example 3.3. Let $K_{1}=\bigcup_{|n| \geq 1}\left(\frac{1}{2} S+2 \pi n\right)$. Then $K_{1} \in \mathcal{K}_{\tau} \backslash \mathcal{K}_{d}$.

Proof. From the definition we see that $K_{1}$ is disjoint with $2 \pi m+\left(-\frac{\pi}{2}, \frac{\pi}{2}\right)$ for every $m \in \mathbb{Z}$. This trivially implies that $K_{1} \in \mathcal{K}_{\tau}$. Next we see that $K_{1} \notin \stackrel{\mathcal{K}_{d}}{\text {. Given any }}$ $\varepsilon>0$ and any large integer $m$ we have

$$
\left|L_{m}^{(\varepsilon)}\right| \geq\left|\bigcup_{\ell=m}^{\infty} \frac{1}{2^{\ell}}\left\{K_{1} \cap 2^{\ell}\left[\frac{\varepsilon}{2}, \varepsilon\right)\right\}\right| \geq 2^{-m}\left|K_{1} \cap 2^{m}\left[\frac{\varepsilon}{2}, \varepsilon\right)\right|
$$

Now, if $m$ is large enough we have

$$
\left|K_{1} \cap 2^{m}\left[\frac{\varepsilon}{2}, \varepsilon\right)\right| \geq \sum_{\substack{n \in \mathbb{Z} \\ 2^{m-1} \varepsilon<2 \pi n<2^{m} \varepsilon-\pi}}\left|2 \pi n+\left[\frac{\pi}{2}, \pi\right)\right| \geq c 2^{m} \varepsilon,
$$

for some constant $c>0$. Thus, $\lim _{m \rightarrow \infty}\left|L_{m}^{(\varepsilon)}\right| \geq c \varepsilon>0$, showing that $K_{1} \notin \mathcal{K}_{d}$.

Example 3.4. Let $K_{2}=\bigcup_{n \geq 1}\left[2^{n} \pi, 2^{n} \pi\left(1+\frac{1}{n}\right)\right)$. Then $K_{2} \in \mathcal{K}_{d} \backslash \mathcal{K}_{\tau}$.

Proof. This time we have, for all $m \geq 1$,

$$
\left|L_{m+1}^{(\pi)}\right|=\left|\bigcup_{\ell=m}^{\infty} \frac{1}{2^{\ell+1}}\left\{K_{2} \cap 2^{\ell+1}\left[\frac{\pi}{2}, \pi\right)\right\}\right|=\left|\bigcup_{\ell=m}^{\infty} \frac{1}{2^{\ell}}\left[2^{\ell} \pi, 2^{\ell} \pi\left(1+\frac{1}{\ell}\right)\right)\right|=\frac{\pi}{m} .
$$

Since this number tends to 0 as $m \rightarrow \infty$, we have established that $K_{2} \in \mathcal{K}_{d}$. On the other hand, for any $\varepsilon>0$ and for integers $m=2^{n}+r$ (with $n$ large enough), we have $\left|K_{2} \cap\left\{2 \pi\left(2^{n}+r\right)+(-\varepsilon, \varepsilon)\right\}\right|=2 \varepsilon$, at least when $0<r<\frac{c 2^{n}}{n}$, for some universal constant $c>0$. This implies

$$
\begin{aligned}
\sum_{m \geq 1} \frac{1}{m}\left|K_{2} \cap\{2 \pi m+(-\varepsilon, \varepsilon)\}\right| & \geq \sum_{n=1}^{\infty} \frac{1}{2^{n+1}} \sum_{0 \leq r<2^{n}}\left|K_{2} \cap\left\{2 \pi\left(2^{n}+r\right)+(-\varepsilon, \varepsilon)\right\}\right| \\
& \geq c \sum_{n=1}^{\infty} \frac{1}{2^{n+1}} \frac{2^{n}}{n} \varepsilon=\infty,
\end{aligned}
$$

and therefore $K_{2}$ cannot belong to $\mathcal{K}_{\tau}$.

\section{Nonadmissibility of $\mathbb{R}$}

In this section we show the following theorem.

Theorem 4.1. For all sets $A \subset \mathbb{R}$ of positive Lebesgue measure we have

$$
\left|\mathbb{R} \backslash \bigcup_{\ell \in \mathbb{Z}} \bigcup_{k \neq 0} 2^{-\ell}(A+k)\right|=0
$$

As a consequence we have $\left[\tau_{\mathbb{R}}(I)\right]=\mathbb{R}$ a.e. for any set $I$ with positive measure. This implies that $\left|\mathcal{L}_{\mathbb{R}}(I)\right|=2 \pi$ for such sets, and therefore, $\mathbb{R} \notin \mathcal{K}\left(J_{0}\right)$ for any bounded Calderón set $J_{0}$. Hence, our results on connectivity do not apply to the full class of PFW's. As we shall see the proof of Theorem 4.1 is not completely 
trivial, and has an unexpected connection with number theory which we shall make clear in the next section. For convenience, throughout these two sections we shall replace the number $2 \pi$ by 1 .

Proof. Set $S=[-1,-1 / 2) \cup[1 / 2,1)$. It suffices to show that

$$
\left|S \backslash \bigcup_{\ell \in \mathbb{Z}} \bigcup_{k \neq 0} 2^{-\ell}(A+k)\right|=0
$$

for all sets $A \subset \mathbb{R}$ of positive Lebesgue measure. By symmetry first and then by rescaling, we may assume with no loss of generality that $A \subset[0,1)$. Moreover, (4.2) will follow from the statement:

For all dyadic intervals $I \subset[0,1)$

$$
\left|[1 / 2,1) \backslash \bigcup_{\ell \geq L+1} \bigcup_{k \geq 1} 2^{-\ell}(I+k)\right|=0, \quad \forall L=0,1,2, \ldots
$$

To see this, given any set $A \subset[0,1)$ of positive Lebesgue measure and $\delta>0$, take a dyadic interval $I$ such that

$$
1-\delta \leq \frac{|I \cap A|}{|I|} \leq 1
$$

By the statement (4.3) with $L=0$ and the nesting property of the family of dyadic intervals $I_{\ell, k}=2^{-\ell}(I+k)$, we can write $[1 / 2,1)$ as a disjoint union $\biguplus_{(\ell, k) \in \mathcal{D}} I_{\ell, k}$ (a.e.), for a certain index set $\mathcal{D} \subset \mathbb{Z}_{+} \times \mathbb{Z}_{+}$. If we denote $A_{\ell, k} \equiv 2^{-\ell}(A+k)$, we also have

$$
\left|[1 / 2,1) \backslash \bigcup_{\ell \geq 1} \bigcup_{k \geq 1} 2^{-\ell}(A+k)\right| \leq \sum_{(\ell, k) \in \mathcal{D}}\left|I_{\ell, k}-A_{\ell, k}\right|=\sum_{(\ell, k) \in \mathcal{D}}\left|I_{\ell, k}\right|-\left|I_{\ell, k} \cap A_{\ell, k}\right| .
$$

Now, by a simple rescaling of (4.4), we see that $\left|I_{\ell, k}\right|-\left|I_{\ell, k} \cap A_{\ell, k}\right| \leq \delta\left|I_{\ell, k}\right|$ and, therefore, we obtain

$$
\left|[1 / 2,1) \backslash \bigcup_{\ell \geq 1} \bigcup_{k \geq 1} 2^{-\ell}(A+k)\right| \leq \sum_{(\ell, k) \in \mathcal{D}} \delta\left|I_{\ell, k}\right|=\delta\left|\biguplus_{(\ell, k) \in \mathcal{D}} I_{\ell, k}\right|=\frac{\delta}{2} .
$$

Since $\delta$ is arbitrary, the left-hand side equals 0 . By symmetry,

$$
\left|(-1,-1 / 2] \backslash \bigcup_{\ell \geq 1} \bigcup_{k \leq-1} 2^{-\ell}(A+k)\right|=0
$$

which gives (4.2). It then remains to prove (4.3).

The result is clearly true for the particular case $I=[0,1)$ since

$$
2^{-(L+1)} \bigcup_{k=2^{L}}^{2^{L+1}-1}(I+k)=2^{-(L+1)}\left[2^{L}, 2^{L+1}\right)=[1 / 2,1) .
$$

The key step to finishing the proof of Theorem 4.1 is contained in the following lemma.

Lemma 4.2. If the inclusion in (4.3) is true for a given dyadic interval $I \subset[0,1)$, then it is true for its two dyadic sons $I^{+}$and $I^{-}, I=I^{+} \cup I^{-}$, and therefore, for all its descendants. 
Proof. Take $L \in \mathbb{N}$. From the hypothesis, there exist positive integers $k_{j}, \ell_{j}$, with $\ell_{j}>L$, so that the intervals $I_{j}=2^{-\ell_{j}}\left(I+k_{j}\right)$ are mutually disjoint, for $j=1,2, \ldots$, and $[1 / 2,1)=\biguplus_{j \geq 1} I_{j}$, a.e. If we set $I_{j}^{ \pm}=2^{-\ell_{j}}\left(I^{ \pm}+k_{j}\right)$, then $\left|I_{j}^{+}\right|=\frac{1}{2}\left|I_{j}\right|$ and so the sequence $\left\{I_{j}^{+}\right\}_{j}$ "covers" $\frac{1}{2}$ of the size of $[1 / 2,1)$. Now fix $j \in \mathbb{N}$ and take $L_{j}=\ell_{j}+1>L$ so that $2^{-L_{j}}|I|=\left|I_{j}^{-}\right|$. Then, from the hypothesis again we can cover $[1 / 2,1)$ by disjoint intervals of the form $2^{-\ell}(I+k)$ with $\ell>L_{j}$. In particular, those intersecting $I_{j}^{-}$are necessarily contained in $I_{j}^{-}$(since they are smaller). Thus, for this $j$, there are positive integers $k_{j, i}, \ell_{j, i}$, with $\ell_{j, i}>L_{j}$, so that the intervals $I_{j, i}=2^{-\ell_{j, i}}\left(I+k_{j, i}\right)$ are mutually disjoint, for $i=1,2, \ldots$, and $I_{j}^{-}=\biguplus_{i \geq 1} I_{j, i}$ a.e. Hence

$$
[1 / 2,1)=\left(\biguplus_{j \geq 1} \biguplus_{i \geq 1} I_{j, i}\right) \biguplus\left(\biguplus_{j \geq 1} I_{j}^{+}\right) .
$$

Call $I_{j, i}^{+}=2^{-\ell_{j, i}}\left(I^{+}+k_{j, i}\right)$. Then $\left\{I_{j}^{+}, I_{j, i}^{+}: j=1,2, \ldots ; i=1,2, \ldots\right\}$ covers $\frac{3}{4}$ of $[1 / 2,1)$. By a straightforward induction process, we obtain the result for $I^{+}$and, similarly, for $I^{-}$.

\section{Connections to number theory}

We begin with an elementary observation. The statement of Theorem 4.1 is equivalent to

(A) There exists a null set $E \subset[0,1)$ so that for all $x \in[0,1) \backslash E$ the sequence of points $\left\{2^{\ell} x(\bmod 1): \ell \geq 1\right\}$ is dense in $[0,1)$.

Suppose (A) holds and let $L \geq 0$ be a fixed integer. If $I$ is any dyadic interval in $[0,1)$ and $x \in[0,1) \backslash E$, by density of $\left\{2^{\ell} x(\bmod 1): \ell>L\right\}$ there must exist integers $\ell>L, k \in \mathbb{N}$ so that $2^{\ell} x-k \in I$. Thus $\left|[0,1) \backslash \bigcup_{\ell>L, k \geq 1} I_{\ell, k}\right|=0$ which implies (4.3). For the converse, if Theorem 4.1 holds we also have

$$
\left|[0,1) \backslash \bigcup_{\ell \geq 1} \bigcup_{k \geq 1} 2^{-\ell}(I+k)\right|=0,
$$

for every dyadic interval $I \subset[0,1)$. Since the number of dyadic intervals is countable, we can define a set of Lebesgue measure zero by

$$
E=\bigcup_{I \text { dyadic }}\left\{[0,1) \backslash \bigcup_{\ell \geq 1} \bigcup_{k \geq 1} 2^{-\ell}(I+k)\right\} .
$$

Then, for every $x \in[0,1) \backslash E$ the sequence $\left\{2^{\ell} x(\bmod 1): \ell \geq 1\right\}$ touches each dyadic interval $I$ (in fact infinitely often), which clearly implies that this sequence must be dense.

The assertion (A) has a number theoretic flavour. In fact, it is actually a consequence of a stronger result related with "uniformly distributed sequences". We say that a sequence $\left\{a_{\ell}\right\}_{\ell=1}^{\infty}$ of real numbers in $[0,1)$ is uniformly distributed in $[0,1)$ if for every interval $I \subset[0,1)$ we have

$$
\lim _{n \rightarrow \infty} \frac{1}{n} \#\left\{1 \leq \ell \leq n: a_{\ell} \in I\right\}=|I| .
$$

Of course, every uniformly distributed sequence must be dense in $[0,1)$. A classical theorem of $\mathrm{H}$. Weyl et al. establishes that for all irrational numbers $x \in[0,1)$ the sequence $\{\ell x\}_{\ell=1}^{\infty}$ is uniformly distributed modulo 1 (see, e.g., [6, Th. 6.3]). There exist several extensions of this result, based on Weyl's celebrated characterization 
of uniformly distributed sequences in terms of limits of exponential sums. One of these extensions establishes that for every strictly increasing sequence $\left\{a_{\ell}\right\}_{\ell=1}^{\infty} \subset \mathbb{N}$ and for a.e. $x \in[0,1)$, the sequence $\left\{a_{\ell} x\right\}_{\ell=1}^{\infty}$ is uniformly distributed modulo 1 (see, e.g., [2, p. 138]). If as a particular case we let $a_{\ell}=2^{\ell}$, then we obtain a stronger version of the result stated in (A).

There is another interpretation of (A) purely in terms of the intrinsic properties of numbers. Indeed if we represent the number $x \in[0,1)$ in its binary expansion, then the fact that the sequence $\left\{2^{\ell} x\right\}_{\ell=1}^{\infty}$ is dense modulo 1 in $[0,1)$ is the same as saying that any arbitrary block of $\{0,1\}$-digits " $b_{1} \ldots b_{k}$ " is contained infinitely often in the binary expansion of $x$. We shall call the numbers $x \in[0,1)$ with this property weakly normal (to base 2). Then, in view of the previous discussion, the statement of Theorem 4.1 is equivalent to saying that a.e. $x \in[0,1)$ is a weakly normal number.

In relation with this concept, a stronger result is also known. We say that a number $x \in[0,1)$ is normal to base $r$ if any arbitrary block made of digits in $\{0,1, \ldots, r-1\}$ of length $k$, say " $B_{k}=b_{1} b_{2} \ldots b_{k}$ ", is contained in the decimal expansion of $x$ in base $r$ with frequency $1 / r^{k}$. That is, if $N\left(B_{k}, n\right)$ counts the number of occurrences of the block $B_{k}$ within the first $n$ digits of the expansion of $x$, then we must have

$$
\lim _{n \rightarrow \infty} \frac{N\left(B_{k}, n\right)}{n}=\frac{1}{r^{k}}, \quad \forall B_{k}, \quad \forall k \geq 1
$$

(see, e.g., [6, Ch. 8] for this and other equivalent definitions). In particular, every normal number to base 2 is weakly normal according to our previous definition. Then, a classical theorem of E. Borel (Rend. Circ. Mat. Palermo 27 (1909), 247271) establishes that a.e. $x \in[0,1)$ is normal to every base $r$. This gives another proof of (A), and hence of Theorem 4.1. In fact, the two notions we have introduced are related by a theorem due to D. Wall which asserts that a number $x \in[0,1)$ is normal to base $r$ if and only if the sequence $\left\{r^{\ell} x\right\}_{\ell=1}^{\infty}$ is uniformly distributed modulo 1 (see [6, Th. 8.15]).

Finally we would like to point out that verifying one of the previous properties for a specific number $x$ can be a very hard question. For instance it is not known whether such numbers as $\sqrt{2}, e$ or $\pi$ are normal to any base. In fact, the set of numbers which are not normal to any base are of course dense (since it contains the rationals) and can be shown to be uncountable [6, p. 116]. As a simple example observe that the binary numbers of the form $x=.0 b_{1} 0 b_{2} 0 b_{3} \ldots$ (for $b_{i}=0,1$ ) are not normal, nor even weakly normal to base 2 . Thus, the exceptional set in assertion (A) is a dense uncountable subset of $[0,1)$.

\section{ACKNOWLEDGEMENTS}

The authors thank Fernando Chamizo and the anonymous referee for useful comments and remarks.

\section{REFERENCES}

1. A. Bonami, S. Durand and G. Weiss, "Wavelets obtained by continuous deformations of the Haar wavelet". Rev. Mat. Iberoam. 12 (1996), 1-18. MR.1387583 (98k:42033)

2. J. Cilleruelo and A. Córdoba, La teoría de los números. Mondadori España (1992). 
3. G. Garrigós, E. Hernández, H. Šikić, F. Soria, G. Weiss and E. Wilson, "Connectivity in the set of Tight Frame Wavelets". Glasnik Matematički. 38 (58) (2003), 75-98. MR.1987107 (2005g:42078)

4. E. Hernández and G. Weiss, A First Course on Wavelets. CRC Press, Boca Raton, FL (1996). MR 1408902 (97i:42015)

5. E. Ionascu, D. Larson and C. Pearcy, "On wavelet sets". J. Fourier Anal. Appl. 4 (1998), 711-721. MR1666001 (2000b:42029)

6. I. Niven, Irrational numbers. The Carus Mathematical Monographs 11. Mathematical Association of America. John Wiley and Sons Inc. (1956). MR0080123 (18:195c)

7. M. Paluszyński, H. Šikić, G. Weiss and Sh. Xiao, "Tight frame wavelets, their dimension functions, MRA tight frame wavelets and connectivity properties". Adv. Comput. Math. 18 (2-4) (2003), 297-327. MR1968123 (2004b:42087)

8. W. Rudin, Real and Complex Analysis, Second Edition, McGraw-Hill (1974). MR0344043 $(49: 8783)$

9. D. Speegle, "The $s$-elementary wavelets are path connected". Proc. Amer. Math. Soc. 127 (1) (1999), 223-233. MR1468204 (99b:42045)

10. The Wutam Consortium, "Basic properties of wavelets". J. Fourier Anal. Appl. 4 (1998), 575-594. MR1658652 (99i:42056)

Departamento de Matemáticas, Universidad Autónoma de Madrid, 28049 Madrid, SPAIN

E-mail address: gustavo.garrigos@uam.es

Departamento de Matemáticas, Universidad Autónoma de Madrid, 28049 Madrid, SPAIN

E-mail address: eugenio.hernandez@uam.es

Department of Mathematics, University of Zagreb, BijeničKa 30, 10000 Zagreb, CroaTIA

E-mail address: hsikic@math.hr

Departamento de Matemáticas, Universidad Autónoma de Madrid, 28049 Madrid, SPAIN

E-mail address: fernando.soria@uam.es 